\title{
Sigmoid CME Source Regions at the Sun: Some Recent Results
}

\author{
Alphonse C. Sterling ${ }^{1}$
}

\section{NASA/Marshall Space Flight Center, SD50/Space Science Department, Huntsville, AL 35812 asterling@solar.stanford.edu}

Journal of Atmospheric and Solar-Terrestrial Physics, 62, 1427 (2000)

${ }^{1}$ NRC_-MSFC Research Associate 


\begin{abstract}
Identifying Coronal Mass Ejection (CME) precursors in the solar corona would be an important step in space weather forecasting, as well as a vital key to understanding the physics of CMEs. Twisted magnetic field structures are suspected of being the source of at least some CMEs. These features can appear sigmoid ( $\mathrm{S}$ or inverse-S) shaped in soft Xray (SXR) images. We review recent observations of these structures and their relation to CMEs, using soft X-ray (SXR) data from the Soft X-ray Telescope (SXT) on the Yohkoh satellite, and EUV data from the EUV Imaging Telescope (EIT) on the SOHO satellite. These observations indicate that the pre-eruption sigmoid patterns are more prominent in SXRs than in EUV, and that sigmoid precursors are present in over $50 \%$ of CMEs. These findings are important for CME research, and may potentially be a major component to space weather forecasting. So far, however, the studies have been subject to restrictions that will have to be relaxed before sigmoid morphology can be used as a reliable predictive tool. Moreover, some CMEs do not display a SXR sigmoid structure prior to eruption, and some others show no prominent SXR signature of any kind before or during eruption.
\end{abstract}

\title{
1 Introduction
}

Coronal Mass Ejections (CMEs) play a major role in the generation of interplanetary disturbances, and can disrupt the magnetic and particle environment near Earth (e.g., Gosling et al. 1991; Webb 1995; McAllister et al. 1996). They are suspected of resulting in damage to satellites in space (e.g., Berdichevsky et al. 1998), while particularly large geomagnetic storms can damage Earth-based power systems, as happened on 1989 March 13 when disruption of a power grid led to a nine-hour power outage in Quebec, Canada (e.g., Joselyn 1998). These, and other "space weather" consequences underline the importance of an improved understanding of CMEs, hopefully leading to an ability to forecast reliably disturbances some time in advance of their occurrence. In addition, CMEs are one of the most dynamic and energetic aspects of solar activity, and explaining their sudden ejection into space poses a formidable problem for solar physicists. Identifying their source regions is a key step in illuminating these physical processes.

CMEs are seen primarily in white-light by using coronagraphs to obscure the bright solar disk, and appear as portions of the enhanced corona expanding away from the Sun. Although visible from some high-altitude ground-based coronagraphs, most data have been obtained with coronagraphs on board satellites. The Skylab mission recorded about $100 \mathrm{CMEs}$ over the period 1973 - 1974, an NRL coronagraph on board the P78-1 mission recorded some 1200 CMEs between 1979 and 1985, and the Solar Maximum Mission (SMM) satellite saw about 1300 events in 1980 and 1984-1989. Most recently, the LASCO coronagraph on the Solar Heliospheric Observatory ( $\mathrm{SOHO}$ ) satellite, which is still in operation, recorded 375 CMEs over a 2.5-year period following its start of operations in January 1996 (Subramanian et al. 1999). Howard et al. (1985) and Hundhausen (1993) describe results from P78-1 and SMM, respectively. Other work reviewing CMEs include papers by Kahler (1992), Gosling (1996), and Low (1996). Hudson \& Webb (1997) review SXR observations, and there are a number of other good discussions in the volume by Crooker et al. (1997).

Mass estimates from the white-light images indicate that CMEs contain $10^{15}-10^{16} \mathrm{~g}$ of material, with reported velocities covering from a few 10 's of $\mathrm{km} \mathrm{s}^{-1}$ to $2000 \mathrm{~km} \mathrm{~s}^{-1}$. Kinetic 
energies associated with the fastest CMEs are $10^{31}-10^{32} \mathrm{erg}$. Their occurrence rate varies in-phase with the solar cycle, ranging from less than one per day to a few per day. These CME properties are based on pre-LASCO data sets, and it will be of value to re-examine these numbers using LASCO, which has better sensitivity and time coverage than the earlier instruments.

One key to understanding - and eventually predicting - CMEs lies with a proper identification of their source regions in the lower solar atmosphere, a task that has been surprisingly difficult. As will be discussed in $\S \S 4$ and 6 below, recent observations from the soft X-ray telescope (SXT) on the Yohkoh spacecraft (Ogawara et al. 1991) indicate that the source of many CMEs appear as long-lived "S"-shaped or "inverse-S" shaped (sigmoid) systems of loops in soft X-ray (SXR) images of the solar corona. If eventually verifiable, such a marker for CME precursors could be both an important key to understanding the origin of CMEs, and a powerful tool for guarding against adverse space weather consequences. In this work we review the observations of these structures and their relation to CMEs, based on data from Yohkoh, launched in 1991, and from the SOHO satellite. We begin with a brief history of sigmoid structures on the Sun from before the era of Yohkoh and SOHO.

\section{Earlier Observations}

Observations in X-rays from Skylab suggested that erupting filaments and two-ribbon flares were associated with twisted magnetic flux tubes or sheared fields (see, e.g., Rust et al. 1980 and Webb 1992 for reviews). CMEs (sometimes referred to as "coronal transients" in earlier work) were recognized to be often related to those phenomena (e.g., Webb 1991). Among the highlights of those studies is that of Kahler (1977), who noted that coronal transients were associated with long-decay-time SXR flares ("long duration events" or "long decay events" - LDEs) in which pre-flare magnetic structures with a non-potential appearance evolved into structures which appeared to be potential during the flare, and then returned to a nonpotential configuration later. He also noted that LDEs usually consist of an arcade of loops. Sheeley et al. $(1975,1983)$ discuss a relation between CMEs and LDEs, while the latter of these papers shows a strong statistical correlation between duration of SXR flares and the likelihood of an associated CME, including a $100 \%$ correlation in their sample for events of duration $\geq 6$ hours.

At times these early observations have suggested sigmoid structures result in CMEs. For example, Sheeley et al. (1976) noted a "sinuous emission feature" which changed form with the suspected ejection of a CME. Due to its superior image quality and improved time coverage, these structures have been seen more readily in Yohkoh SXT data. Examples include Sakurai et al. (1992), Manoharan et al. (1996), Rust \& Kumar (1996), and Moore et al. (1997). Rust \& Kumar (1996) and Rust (1997) described these structures as sigmoid because of their shape. Nakagawa et al. (1971) described how the shapes could come about in force-free fields in filaments. These earlier SXT observations, however, were not positively associated with CMEs as there was no space-borne coronagraph available at that time prior to the start of $\mathrm{SOHO}$ operations in 1996.

Meanwhile, observations in interplanetary space reveled "magnetic clouds," in which the magnetic fields of disturbances related to ejections from the Sun show a characteristic signature of the field of a flux rope (e.g., Burlaga et al. 1981; Marubashi 1986). Moreover, 
bidirectional streaming (also referred to as counterstreaming, e.g., Gosling 1996) of particles trapped in these magnetic clouds indicated that these fields were rooted in the surface of the Sun. Reviews of magnetic clouds include Burlaga (1984, 1991, 1995), and Osherovich and Burlaga (1997). Chen et al. (1997) present evidence from SOHO data that a flux rope results in a CME.

Studies such as these strongly suggested a relation between twisted or sheared magnetic fields and erupting or explosive structures. This connection has been explored extensively with efforts ranging from cartoon descriptions to detailed analytical investigations. Early examples include Rust (1976) and Sakurai (1976), while more recent theoretical work includes Chen (1989; 1996); Forbes (1990); Antiochos et al. (1994, 1999); Rust \& Kumar (1994, 1996); Rust (1997); Aulanier et al. (1998); and Matsumoto et al. (1998).

\section{Yohkoh SXT and SOHO EIT Instruments}

Yohkoh has observed the Sun from Earth orbit nearly continuously since August 1991. SXT, one of four Yohkoh instruments, produces broad-band SXR images with a CCD camera with 2.5 "-pixels. It utilizes five analysis filters of varying thicknesses and transmission characteristics, allowing for determination of plasma properties such as electron temperatures and emission measures. These filters cover wavelengths ranging from approximately $3-45 \AA$, and are most responsive to plasmas of temperatures in excess of $2-3 \mathrm{MK}$. Typically SXT takes several full-disk images each Yohkoh orbit $(\approx 96 \mathrm{~min})$. A major advantage of SXT over previous space-borne X-ray telescopes is its high time cadence and continuity of operation. Tsuneta et al. (1991) provide details of the SXT instrument.

In contrast to Yohkoh, SOHO is located at the L1 Lagrangian point, allowing for solar observations uninterrupted by a day-night cycle. Among its suite of instruments, two have been particularly key to CME imaging studies, the LASCO coronagraphs and the EUV Imaging Telescope (EIT). LASCO is a combination of three coronagraphs, C1, C2, and C3, with fields of view of $1.1-3,2-6$, and $3.5-30$ solar radii, respectively. C1 observes in the $5303 \AA$ Fe XIV line, while C2 and C3 are white-light coronagraphs. Brueckner et al. (1995) describe the instrument in more detail.

EIT is a multi-layer Ritchey-Chretien telescope with several filters and a CCD detector. It produces full-Sun images with a spatial resolution of 2.6". The $195 \AA$ Fe XII filter has been used most extensively for CME observations. It has a narrow-band peak response to plasmas of approximately 1.5 MK. Delaboudiniere et al. (1995) provide more details of EIT.

As indicated in the Introduction, there were no space-based CME observations available between the end of the SMM mission in 1989 and the LASCO observations from SOHO beginning in late 1996. Consequently, for the first five years of the Yohkoh mission, detecting CMEs was problematic, making it even more difficult to identify reliably CME source regions. Thus the combined observations from LASCO, to identify CMEs, with SXT and EIT for imaging the hotter and cooler corona, respectively, gives us unprecedented opportunities to study CME coronal sources. 


\section{Yohkoh SXT Observations of CME Source Regions}

Several of the CME studies with SXT have focused on "halo CMEs." These are CMEs which appear to surround or partially surround the coronagraph occulting disk, indicating that the material is ejected either toward or away from the Earth. The first such event was reported by Howard et al. (1982) using P78-1 data. Halo CMEs are particularly useful for studying CME coronal sources, since their source regions are expected to be near the center of the solar disk for those directed toward the Earth.

One of the earliest halo CMEs identified in the LASCO data occurred on 1997 April 7. Sterling \& Hudson (1997) examined SXT data for that event. They found that initiation of the CME closely coincided with a soft X-ray flare. For at least two days prior to the $\mathrm{CME}$, the active region in which the flare occurred consisted of the sigmoid structure shown in Figure 1a. During the flare, this structure reconfigured to form a cusp-shaped structure (Fig. 1b). Figure 1c shows a difference image, formed by subtracting the pre-event image in (1a) from the post-event image in (1b); locations that had been occupied by the sigmoid appear dark in (1c). Sterling \& Hudson calculated that the reduced-intensity area in the difference image, called "dimming regions," correspond to a mass of at least $10^{14}$ g having been ejected from the Sun. This is one or two orders of magnitude less than the mass typically associated with CMEs, indicating that the dimming areas seen on the disk account for only a small amount of the CME mass in this case. This assumes that the dimmings are due to mass loss, rather than a reduction in temperature of the material - an assumption supported by EIT observations (see $\S 5$ ).

For an event occurring near the limb, however, Gopalswamy \& Hanaoka (1998) observed dimmings in SXT images corresponding to $10^{15} \mathrm{~g}$, which is typical of a CME mass. They may have observed a larger mass loss than did Sterling \& Hudson because most of their dimmed region was of material beyond the limb, rather than projected on the solar disk where surrounding features may complicate the analysis. Or it could be that they were observing a larger CME than did Sterling \& Hudson. Whichever the case, the Gopalswamy \& Hanaoka work provides additional confirmation that dimmings can be a result of the expulsion of a CME.

Dimmings on the disk, as in the case of Sterling \& Hudson (also see Hudson et al. 1996; Hudson \& Webb 1997), also include the "transient coronal holes" (e.g., Rust 1983; Watari et al. 1995; Kozuka et al. 1995; Manoharan et al. 1996; Smith et al. 1997) as categorized by Hudson \& Webb (1997). These structures do qualitatively resemble coronal holes seen in the polar regions in SXRs, although Sterling \& Hudson could not determine if the intensity of the dimming regions was as low as that of coronal holes with the data they used.

Hudson et al. (1998) extended the Sterling \& Hudson work to cover halo CMEs observed between 1996 December and 1997 June. This was a particularly useful period for observing CME source regions, being just beyond solar minimum so that there was a substantial number of active regions, but not so many that following individual regions was difficult. Hudson et al. (using data compiled by C. St. Cyr 1997, private communication) found 11 halos during this period. They determined that six or seven of these 11 originated on the Earthward-side of the Sun (data coverage with SXT was too poor to make a determination for an event near 00:31 UT on 1997 April 27). Webb et al. (2000) also examined the halo CMEs during this same time period. They added three additional halo CMEs that had not been found in the data at the time of the Hudson et al. work, and one of these (occurring on 1997 March 9) was 
a front-side event. Webb et al. also concluded that the 00:31 UT 1997 April 27 event most likely was a back-side event. Thus, among a total of 14 confirmed halos over the six-month period, about 7 originated from the near-side of the Sun. This indicates that visibility of halos does not strongly depend on whether they are directed toward or away from Earth.

Among the six front-side events seen in SXT by Hudson et al. (1998), four of them, occurring on 1996 December 19; 1997 April 7; 1997 May 12; and 1997 May 21, had the "sigmoid-to-arcade" characteristics described by Sterling \& Hudson (1997), whereby a preflare sigmoid transforms into a cusp or arcade of loops in the post-flare phase, and there is associated coronal dimming (although these characteristics were only marginal in the May 21 event; see Sterling et al. 2000). A fifth event, occurring on 1997 February 7, was qualitatively different in SXRs from those four in that it was associated with a large-scale coronal arcade rather than primarily a lone active region. The remaining event (of 1997 January 6) differed from the other five in that it had an extremely weak signature in SXRs. It would have escaped notice were it not for the identification of the halo $\mathrm{CME}$, and its detection in interplanetary space and geophysical consequences (e.g., Burlaga et al. 1998; Fox et al. 1998; Reiner et al. 1998; Watari \& Watanabe 1998; Webb et al. 1998). Thus, although overall the Hudson et al. (1998) results suggest that the sigmoid-to-arcade pattern for CME source regions is not uncommon, the January 6 event demonstrates the existence of some geophysically important events that occur without any accompanying clear signature in SXRs.

Hudson et al. (1998) did not investigate details of how the transformation between preflare sigmoid and post-flare cusp occurred. Manoharan et al. (1996), however, studied such evolution for a sigmoid-to-arcade event (although not using this terminology) of 1994 October 25, using SXT and Nancay radioheliograph data. They found that first the sigmoid expanded, and later the cusped arcade formation started around the central part of the expanding sigmoid.

\section{SOHO EIT Observations of CME Source Regions}

There are several published reports on SOHO EIT observations related to CMEs (e.g., Dere et al. 1997; Aurass et al. 1999; Innes et al. 1999). We restrict our discussion here primarily to events connected with sigmoid structures seen in SXT.

Zarro et al. (1999) combined EIT observations with SXT images of the 1997 April 7 event. They found that, unlike in SXRs, a distinct sigmoid structure is not apparent in the EIT pre-flare images; rather, a more complex system of loops is visible. In the post-flare period, EIT images show a system of loops at the location of the SXR cusp. Zarro et al. also found dimming to be present in the EIT images at the same locations as those seen in SXT. Since the EIT images are at a cooler temperature than SXT, the appearance of coincident dimmings in SXT and EIT implies that they are likely due to a density reduction, rather than to a reduction in temperature of the material. In the latter case, a brightening in EIT images would be expected as the material cools from SXR temperatures to EUV temperatures. Thompson et al. (1998) discuss dimmings seen in EIT for the 1997 May 12 event.

Sterling et al. (2000) extended the EIT-SXT comparisons of Zarro et al. to the four events displaying sigmoid-to-arcade characteristics (including the 1997 April 7 event) found by Hudson et al. (1998) discussed in $§ 4$. They confirmed the Zarro et al. results, finding 
that prior to eruption the sigmoid pattern is more prominent in the SXT image than in the EIT $195 \AA$ images. They also found, however, that for a short period during eruption the sigmoid feature was prominent in EUV also. The pre-event sigmoid shape is more prominent in the hotter $(2.0 \mathrm{MK})$ EIT $284 \AA$ filter than the EIT $195 \AA$ filter, but it is most prominent in the $\gtrsim 3 \mathrm{MK}$ SXT images. This implies that the sigmoid structure is a high-temperature feature (i.e., the plasma entrained in the sheared sigmoid fields is strongly heated). Figure 2 compares SXT and EIT $195 \AA$ pre- and post-flare images for the 1996 December 19 event. Images such as these show that the central portions of the sigmoid structures appear highly sheared and tend to follow the magnetic neutral line (using magnetograms from the MDI instrument on $\mathrm{SOHO}$ ), and that the sigmoid feature's "feet" are rooted in locations distant from the roots of the post-flare cusp feature.

Sterling et al. (2000) summarized the above findings in the schematic shown in Figure 3. This picture is very similar to that describing erupting prominences and two-ribbon flares by Hirayama (1974), Heyvaerts et al. (1977), Moore \& LaBonte (1980), Shibata et al. (1995), Moore et al. (1997), and several others. Future examination of data from various instruments should help differentiate between predictions of various models. For example, it is still to be clarified whether the sigmoid structure is truly an S-shaped flux rope rooted in the surface only at its ends (e.g., Rust \& Kumar 1996), or if it is composed of two interlocking c-shaped structures whose reconnection drives the eruption (e.g., Pevtsov et al. 1996).

van Driel-Gesztelyi et al. (2000) also discuss the non-potentiality of the magnetic field associated with the sigmoid features, and deal with the sigmoid-CME relationship. They show another example of a sigmoid running parallel to the magnetic inversion line in the middle part of an inverse-S, and curling away from the inversion line at both ends, as sketched in Figure 3.

Another feature sometimes seen in EIT $195 \AA$ images in association with CMEs is a large-scale disturbance emanating from flaring regions. These "EIT waves" are described for the 1997 April 7 and 1997 May 12 events by Thompson et al. (1999) and Thompson et al. (1998), respectively. They were also seen in the 1997 May 21 event (Webb et al. 2000). EIT's cadence was too low to make such an identification of these waves prior to 1997 March, meaning that the waves were associated with each of the front-side halo CMEs discussed in Hudson et al. for which observations were possible (Webb et al. 2000). These waves are also seen in association with a partial halo CME of 1997 September 24 (Torsti et al. 1999), while Dere et al. (1997) report a wave emanating from the apparent source region of a non-halo CME originating near the limb.

EIT waves typically have speeds of a few hundred $\mathrm{km} \mathrm{s}^{-1}$. They may be an EUV counterpart to the chromospheric phenomena known as "Moreton waves" (Athay \& Moreton 1961), although this identification is still not certain. Uchida $(1968,1974)$ and Uchida et al. (1973) have suggested that the Moreton waves are the consequence of the propagation of a fast-mode shock wave. Radio data also suggest that these may be shock waves (Kaiser et al. 1998). A similar wave phenomenon has not been convincingly seen in SXT images. This may be a consequence of poor cadence of full-disk images taken during flare times, or the waves may be less prominent in SXRs due to some other factor (Sterling \& Hudson 1997; J. R. Lemen 1997, private communication). Faint hints of such a wave may have been seen with an SXR telescope on Skylab (Rust \& Svestka 1979), which had a sensitivity similar to that of SXT, but extending to slightly longer wavelengths (2-54 A; Sterling \& Hudson used SXT 
images covering approximately $3-20 \AA$ ). Skylab also did not have high time cadence, and thus the question of the visibility of EIT waves at SXR wavelengths is still an outstanding issue.

\section{Sigmoid Connection to Eruptive Events: Statistics}

Although based on a small number of events, Hudson et al.'s (1998) study suggests that a large fraction of the SXR source regions of halo CMEs have pre-flare sigmoid shape. But given a sigmoid structure on the Sun, how likely is it to produce a CME? Canfield et al. (1999) explored this question with a statistical study. They examined Yohkoh SXT movies during two years: 1993 and 1997. These two years were selected because they were periods between solar maximum and minimum; thus there were enough active regions on the Sun to carry out their study, yet not so many that individual regions were confused with neighboring regions. They selected active regions which they were able to follow and observe clearly for an extended period of time, 117 in all. They classified each of these active region as "sigmoidal" if it appeared S or inverse-S shaped, or "non-sigmoidal" if there was no such appearance. Although this judgment was qualitative, it was made independently by two of the workers. They also noted the sunspot area of each region. Finally, they identified each of the 117 region as "eruptive" or "non-eruptive," where eruptive means that they saw formation of transient X-ray loops of either cusped or arcade form. Once again, this judgment was made independently by two workers.

They found that: (i) sigmoidal regions have a tendency to be more eruptive than do non-sigmoidal regions, (ii) this tendency increases with region size, and (iii) for the largest regions (sunspot area $>200$ millionths of a hemisphere, amounting to 14 of the active regions in their study) all of the regions were eruptive, whether sigmoidal or not. Among all of their data, $52 \%$ (61 out of 117) were sigmoidal, and that $52 \%$ accounts for $65 \%$ of the eruptions. Of all the sigmoidal regions, $84 \%$ were eruptive, whereas $50 \%$ of the non-sigmoidal regions were eruptive. Thus, sigmoidal regions are more likely to be eruptive than non-sigmoidal regions, and large regions are more likely to be eruptive than smaller regions.

There are some qualifications on the findings of this important work by Canfield et al. First, they did not utilize coronagraph data in defining eruptive signature, and therefore it is not certain how many of the eruptive events actually resulted in CMEs. As discussed in $\S \S 2,4$, and 5, when a CME is observed, arcade formations (including cusped arcades) are often seen in the low corona. But there may be some percentage of cases where arcades form without a CME. Nonetheless, work such as that of Kahler (1977) linking arcades with LDEs, and Sheeley et al. (1983) linking LDEs and CMEs, suggests that many of the eruptive events seen by Canfield et al. did result in CMEs. A second qualification is that they only considered active regions with a NOAA classification, whereas CMEs can originate from quieter regions also (e.g., McAllister et al. 1996). A third qualification is the subjective determination of the morphology of the regions. These qualifications should be addressed in future studies.

A possible explanation for points (ii) and (iii) listed above for the Canfield et al. results is that sheared fields (characterized by sigmoidal structure) may be "hidden" inside of larger regions, and so such regions may be classified as non-sigmoidal even though they contain a large amount of sheared fields. A quantitative measure of the amount of shear in a region may tighten the statistics even further. Such a study would also be useful in clarifying the 
eruptive mechanism.

If the connection between sigmoid morphology and CMEs discussed by Hudson et al., and the connection between sigmoidal morphology-plus-surface area of regions and their tendency to erupt discussed by Canfield et al., continue to be supported by further study, then potentially this information alone (i.e., region sigmoid morphology and surface area) will provide a relatively simple method for predicting which structures will generate CMEs. The above studies suggest that this method would be able to predict CMEs from sigmoid structures with a confidence level well in excess of $50 \%$. Even if subsequent studies increasing the event sample of Hudson et al. and addressing the qualifications of the Canfield et al. study reduce this confidence level to about $50 \%$, this method could be useful for a variety of purposes, ranging from planning CME or magnetospheric observations with spacecraft to minimizing risks for space travelers planning EVAs on interplanetary space missions.

\section{Conclusions}

Twisted magnetic structures have long been associated with filament eruptions and tworibbon flares. Of late their connection with ejection of CMEs has received increased attention, and images from Yohkoh and $\mathrm{SOHO}$ have shed new light on these structures. To date, the observations show or suggest that: (i) sigmoid structures are often seen in SXRs prior to the onset of CMEs; (ii) these sigmoid structures are more apparent in SXT (SXR) images than EIT (EUV) images, indicating that they are hotter features than typically seen in EUV; (iii) regions sigmoid prior to eruption evolve into un-sheared arcades or cusps after eruption; (iv) footpoints of the pre-eruption features are different from footpoints of post-eruption features; (v) dimming regions associated with the sigmoid-feature eruptions are seen in both SXRs and EUV, they are likely due to density depletions, and the associated mass loss from SXR dimming seen on the disk is an order of magnitude (or more) less than the mass of a typical CME. Mass losses corresponding to dimming in EIT images have yet to be calculated.

Another significant finding is that there are also clear examples of front-side CMEs that are not associated with sigmoid signatures, and sometimes they have virtually no signature in SXRs at all. In the Hudson et al. (1998) study, three of six events were clearly sigmoid, one was marginally sigmoid, one was not "simply" sigmoid, and one had no significant SXR manifestation of any kind.

It is possible, however, that the differences between these latter two events (of 1997 January 6 and 1997 February 7) and the former four sigmoid-to-arcade events of Hudson et al. may only be apparent. That is, sigmoid-related CMEs which involve filament eruptions situated in decaying or decayed active regions with dispersed magnetic field may have very weak SXR signature compared to the ones which occur in young active regions with strong magnetic field concentrations, although they may imply the same physics. Thus events such as those of January 6 and February 7 may represent exceptions from a purely morphological point of view only. This possibility should be examined in future studies.

Most of the results discussed here are based on observations related to halo CMEs. There is, however, conflicting evidence on whether halo CMEs are typical of all CMEs (Webb et al. 2000); for example, only the more energetic CMEs may be bright enough to appear as halos. Still, studies by Bruckner et al. (1998) and Webb et al. (2000) indicate that halo CMEs are preferentially geo-effective, and so even at the $50 \%$ level, observations of sigmoid 
structures are potentially useful in space weather prediction.

Finally, the question of when a sigmoid will erupt has not yet been addressed, but it may eventually be possible to do so with quantitative measurements of magnetic shear values. Another outstanding question is whether the sigmoid regions are the sources for the entire CME, or for only one portion of the the structure.

The bulk of this work was performed while the author held a National Research CouncilNASA/MSFC Research Associateship. A portion of this study was supported by the NRL/ONR basic research programs. A version of this work was presented as an invited talk at the 22nd General Assembly of the International Union of Geodesy and Geophysics (IUGG), held at the University of Birmingham, UK, in July 1999. The author thanks the SCOSTEP committee for partial financial support for the meeting. He also thanks G. A. Gary, H. S. Hudson, R. L. Moore, and an anonymous referee for helpful comments and suggestions.

\section{References}

Antiochos, S. K., Dahlburg, R. B., Klimchuk, J. A. 1994. The magnetic field of solar prominences. Astrophys. J., 420, L41-L44.

Antiochos, S. K., Devore, C. R., Klimchuk, J. A. 1999. A model for solar coronal mass ejections. Astrophys. J., 510, 485-493.

Athay, R. G., Moreton, G. E. 1961. Impulsive phenomena of the solar atmosphere. I. Some optical events associated with flares showing explosive phase. Astrophys. J., 133, 935945.

Aulanier, G., Demoulin, P., van Driel-Gesztelyi, L., Mein, P, DeForest, C. 1998. 3-d magnetic configurations supporting prominences: II. The lateral feet as a perturbation of a twisted flux-tube. Astron. Astrophys., 335, 309-322.

Aurass, H., Vourlidas, A., Andrews, M. D., Thompson, B. J., Howard, R., H., Mann, G. 1999. Nonthermal radio signatures of coronal disturbances with and without coronal mass ejections. Astrophys. J., 511, 451-465.

Berdichevsky et al. 1998. Evidence for Multiple Ejecta: April 7-11, 1997, ISTP Sun-Earth Connection Event. Geophys. Res. Lett., 25, 2473.

Brueckner, G. E., et al. 1995. The large angle spectroscopic coronagraph (LASCO). Solar Phys., 162, 357-402.

Brueckner, G. E., et al. 1998. Geomagnetic storms caused by coronal mass ejections (CMEs): March 1996 through June 1997. Geophys. Res. Lett., 25, 3019-3022.

Burlaga, L. F., Sittler, E., Mariani, R., Schwenn, R. 1981. Magnetic loop behind an interplanetary shock: Voyager, helios and IMP-8 observations. J. Geophys. Res., 86, 6673-6684.

Burlaga, L. F. 1984. MHD processes in the outer heliosphere. Space Sci. Rev., 39, 255-316.

Burlaga, L. F. 1991. Magnetic clouds. In: Physics of the inner heliosphere, vol. 2, R. Schwenn, E. Marsch (eds.), (Springer-Verlag, New York, 1991), 1-22.

Burlaga, L. F. 1995. Interplanetary magnetohydrodynamics. Oxford Univ. Press (New York: 1995).

Burlaga, L., et al. 1998. A magnetic cloud containing prominence material: January 1997. J. Geophys. Res., 103, 277-285.

Canfield, R. C., Hudson, H. S., McKenzie, D. E. 1999. Sigmoidal morphology and eruptive 
solar activity. Geophys. Res. Lett., 26, 627-630.

Chen, J. 1989. Effects of toroidal forces in current loops embedded in a background plasma. Astrophys. J., 338, 453-470.

Chen, J. 1996. Theory of prominence eruption and propagation: Interplanetary consequences. J. Geophys. Res., 101, 27,499-27,519.

Chen, J., et al. 1997. Evidence of an erupting magnetic flux rope: LASCO coronal mass ejection of 1997 April 13. Astrophys. J., 490, L191-L194.

Crooker, N., Joselyn, J., Feynman, J. (eds.) 1997. Coronal mass ejections. Geophys. Monogr. Ser., vol. 99 (AGU: Washington D. C.).

Delaboudiniere, J.-P. et al. 1995. EIT: Extreme-ultraviolet imaging telescope for the soho mission. Solar Phys., 162, 291-312.

Dere, K. P. et al. 1997. EIT and LASCO observations of the initiation of a coronal mass ejection. Solar Phys., 175, 601-612.

Forbes, T. G. 1990. Numerical simulation of a catastrophe model for coronal mass ejections. J. Geophys. Res., 95, 11,919-11,931.

Fox, N. J., Peredo, M., Thompson, B. J. 1998. Cradle-to-grave tracking of the January 6-11, 1997, Sun-Earth connection event. Geophys. Res. Lett., 25, 2461-2464.

Gopalswamy, N., Hanaoka, Y. 1998. Coronal dimming associated with a giant prominence eruption. Astrophys. J., 498, L179-L182.

Gosling, J. T. 1996. Corotating and transient solar wind flows in three dimensions. Annu. Rev. Astron. Astrophys., 34, 35-74.

Gosling, J. T., McComas, D. J., Phillips, J. L., \& Bame, S. J. 1991. Geomagnetic activity associated with Earth passage of interplanetary shock disturbances and coronal mass ejections. J. Geophys. Res., 96, A5, 7831-7839.

Heyvaerts, J., Priest, E. R., Rust, D. M. 1977. An emerging flux model for the solar flare phenomenon. Astrophys. J., 216, 123-137.

Hirayama, T. 1974. Theoretical model of flares and prominences. I: Evaporating flare model. Solar Phys., 34, 323-338.

Howard, R. A., Michels, D. J., Sheeley, N. R., \& Koomen, M. J. 1982. The observation of a coronal transient directed at Earth. Astrophys. J., 263, L101-L104.

Howard, R. A., Sheeley, N. R., Jr., Michels, D. J., Koomen, M. J. 1985. Coronal mass ejections: 1979-1981. J. Geophys. Res., 90, 8173-8191.

Hudson, H. S., Acton, L. W., Freeland, S. L. 1996. A long-duration solar flare with mass ejection and global consequences. Astrophys. J., 470, 629-635.

Hudson, H. S., Webb, D. F. 1997. Soft X-ray signatures of coronal ejections. In: Geophysical Monographs No. 99, "Coronal Mass Ejections," eds. N. Crooker, J. Joselyn, \& J. Feynman, AGU, Washington, p. 27-38.

Hudson, H. S., Lemen, J. R., St. Cyr, O. C., Sterling, A. C., Webb, D. F. 1998. X-ray coronal changes during halo CMEs. Geophys. Res. Lett., 25, 2481-2484.

Hundhausen, A. J. 1993. Sizes and locations of coronal mass ejections: SMM observations from 1980 and 1984-1989. J. Geophys. Res., 98, no. A8, 13,177-13,200.

Innes, D. E., et al. 1999. Multi-wavelength observations of the onset phase of a coronal mass ejection. Solar Phys., 186, 337-361.

Joselyn, J. 1998. The human impact of solar flares and magnetic storms. In: From the Sun: Auroras, Magnetic Storms, Solar Flares, Cosmic Rays, S. T. Suess \& B. T. Tsurutani 
(eds.), AGU (Washington, D. C.), 67-72.

Kahler, S. 1977. The morphological and statistical properties of solar X-ray events with long decay times. Astrophys. J., 214, 891-897.

Kahler, S. W. 1992. Solar flares and coronal mass ejections. Annu. Rev. Astron. Astrophys., 30, $113-141$.

Kaiser, M. L., Reiner, M. J., Gopalswamy, N., Howard, R. A., St. Cyr, O. C., Thompson, B. J., Bougeret, J.-L. 1998. Type II radio emissions in the frequency range from 1-14 MHz associated with the April 7, 1997 solar event. Geophys. Res. Lett., 25, 2501-2504.

Kozuka, Y., Watanabe, T., Kojima, M., Ohyama, M., Tsuneta, S., Khan, J. I., Watari, S. 1995. The dynamical characteristics of a disappearing-filament associated interplanetary disturbance observed in 1992 early May. Pub. Astro. Soc. Japan, 47, 377-381.

Low, B. C. 1996. Solar activity and the corona. Solar Phys., 167, 217-265.

Manoharan, P. K., van Driel-Gesztelyi, L., Pick, M., \& Demoulin, P. 1996. Evidence for large-scale solar magnetic reconnection from radio and X-ray measurements. Astrophys. J., 468, L73-L76.

Marubashi, K. 1986. Structure of the interplanetary magnetic clouds and their origins. Adv./ Space Res., 6(6), 335-338.

Matsumoto, R., Tajima, T., Chou, W., Okubo, A., Shibata, K. 1998. Formation of a kinked alignment of solar active regions. Astrophys. J., 493, L43-L46.

McAllister, A. H., Dryer, M., McIntosh, P., Singer, H., \& Weiss, L. 1996. A large polar crown CME and a "problem" geomagnetic storm: April 14-23, 1994. J. Geophys. Res., 101, $13,497-13,511$.

Moore, R. L., LaBonte, B. J. 1980. The filament eruption in the 3B flare of July 29, 1973: onset and magnetic field configuration. In: Solar and interplanetary dynamics, IAU Symp. 91. M. Dryer, E. Tandberg-Hanssen (eds.) (Reidel: Boston), 207-210.

Moore, R. L., Schmieder, B., Hathaway, D. H., Tarbell, T. D. 1997. 3-d magnetic field configuration late in a large two-ribbon flare. Solar Phys., 176, 153-169.

Nakagawa, Y., et al. 1971. On the topology of filaments and chromospheric fibrils near sunspots. Solar Phys., 19, 72-85.

Ogawara, Y., Takano, T., Kato, T., Kosugi, T., Tsuneta, S., Watanabe, T., Kondo, I., Uchida, Y. 1991. The solar-A mission - an overview. Solar Phys., 136, 1-16.

Osherovich, V. A., Burlaga, L. F. 1997. Magnetic clouds. In: Coronal mass ejections, Geophys. Monogr. Ser., vol. 99, N. Crooker, J. Joselyn, J. Feynman (eds.) (AGU: Washington D. C.), $157-168$.

Pevtsov, A., Canfield, R. C., Zirin, H. 1996. Reconnection an helicity in a solar flare. Astrophys. J., 473, 533-538.

Reiner, M. J., Kaiser, M. L., Fainberg, J., Bougeret, J.-L., Stone, R. G. 1998. On the origin of radio emissions associated with the January $6-11,1997$, CME. Geophys. Res. Lett., $25,2493-2496$.

Rust, D. M. 1976. An active role for magnetic fields in solar flares. Solar phys., 47, 21-40.

Rust, D., M. 1997. Magnetic helicity, MHD kink instabilities and reconnection in the corona. In: Magnetic Reconnection in the Solar Atmosphere. ASP Conference Series; Vol. 111; 1997; ed. R. D. Bentley and J. T. Mariska (1997), 353-358.

Rust, D. M., Svestka, Z. 1979. Slowly moving disturbances in the X-ray corona. Solar Phys. $63,279-295$. 
Rust, D. M., et al.. 1980. Mass ejections. In: Solar Flares: A monograph from Skylab solar workshop II, P. A. Sturrock (ed.). Colorado Associated university Press (Bolder 1980), $273-339$.

Rust, D. M. 1983. Coronal disturbances and their terrestrial effects: Tutorial lecture. Space Sci. Rev., 34, $21-36$.

Rust, D., M., Kumar, A. 1994. Helical magnetic fields in filaments. Solar Phys. 155, 69—97.

Rust, D., M., Kumar, A. 1996. Evidence for helically kinked magnetic flux ropes in solar eruptions. Astrophys. J., 464, L199-L202.

Sakurai, T. 1976. Magnetohydrodynamic interpretation of the motion of prominences. Publ. Astron. Soc. Japan, 28, 177-198.

Sakurai, T., Shibata, K., Ichimoto, K., Tsuneta, S., Acton, L. 1992. Flare-related relaxation of magnetic shear as observed with the Soft X-ray Telescope of Yohkoh and with vector magnetographs. Pub. Astro. Soc. Japan, 44, L123-L127.

Sheeley, N. R., et al. 1975. Coronal changes associated with a disappearing filament. Solar Phys., 45, 377-392.

Sheeley, N. R., Howard, R. A., Koomen, M. J., Michels, D. J. 1983. Associations between coronal mass ejections and soft X-ray events. Astrophys. J., 272, 349-354.

Shibata, K., Masuda, S., Shimojo, M., Hara, H., Yokoyama, T., Tsuneta, S., Kosugi, T., Ogawara, Y. 1995. Hot-plasma ejections associated with compact-loop solar flares. Astrophys. J., 451, L83-L85.

Smith, Z., Watari, S., Dryer, M., Manoharan, P. K., McIntosh, P. S. 1997. Identification of the solar source for the 18 October 1995 magnetic cloud. Solar Phys., 171, 177-190.

Sterling, A. C., Hudson, H. S. 1997. Yohkoh SXT observations of X-ray "dimming" associated with a halo coronal mass ejection. Astrophys. J., 491, L55-L58.

Sterling, A. C., Hudson, H. S., Thompson, B. J., Zarro, D. M. 2000. Yohkoh SXT and SOHO EIT observations of "sigmoid-to-arcade" evolution of structures associated with halo CMEs. Astrophys. J., in press.

Subramanian, P., Dere, K. P., Rich, N. B., \& Howard, R. A. 1999. The relationship of coronal mass ejections to streamers. J. Geophys. Res., 104, 22,321-22,330.

Thompson, B. J., Plunkett, S. P., Gurman, J. V., Newmark, J. S., St. Cyr, O. C., Michels, D. J. 1998. SOHO/EIT observations of an Earth-directed coronal mass ejection on May 12, 1997. Geophys. Res. Lett., 25, 2461-2464.

Thompson, B. J., et al. 1999. SOHO/EIT observations of the 1997 April 7 coronal transient: Possible evidence of coronal Moreton waves. Astrophys. J., 517, 151L-L154.

Torsti, J., Kocharov, L. G., Teittinen, M., Thompson, B. J. 1999. Injection of $\gtrsim 10 \mathrm{MeV}$ protons in association with a coronal Moreton wave. Astrophys. J., 510, 460-465.

Tsuneta, S., et al. 1991. The soft X-ray telescope for the Solar-A mission. Solar Phys., 136, $37-67$.

Uchida, Y. 1968. Propagation of hydromagnetic disturbances in the solar corona and Moreton's wave phenomenon. Solar Phys., 4, 30-44.

Uchida, Y., Altschuler, M. D., Newkirk, G. 1973. Flare-produced coronal MHD-fast-mode wavefronts and Moreton's wave phenomenon. Solar Phys., 28, 495-516.

Uchida, Y. 1974. Behavior of the flare produced coronal MHD wavefront and the occurrence of type II radio bursts. Solar Phys., 39, 431-449.

L. van Driel-Gesztelyi, L., et al. 2000. Initiation of CMEs: the role of magnetic shear and 
helicity. J. Atm. Solar-Terr. Phys., submitted.

Watari, S., Kozuka, T., Ohyama, M., Watanabe, T. 1995. Soft X-ray coronal holes observed by the Yohkoh SXT. J. Geomagn. Geoelectr., 47, 1063-1071.

Watari, S., Watanabe, T. 1998. The solar drivers of geomagnetic disturbances during solar minimum. Geophys. Res. Lett., 25, 2489-2492.

Webb, D. F. 1992. The solar sources of coronal mass ejections. In: Eruptive Solar Flares, Edited by Z. Svestka, B. V. Jackson, \& M.E. Machado (Springer-Verlag: Berlin 1992), $234-247$.

Webb, D. F. 1995. Coronal mass ejections: The key to major interplanetary and geomagnetic disturbances. Reviews of Geophys. Supplement, U.S. National Report to International Union of Geodesy and Geophysics 1991-1994, 577-583.

Webb, D. F., Cliver, E. W., Gopalswamy, N., Hudson, H. S., St. Cyr, O. C. 1998. The solar origin of the January 1997 coronal mass ejection, magnetic cloud and geomagnetic storm. Geophys. Res. Lett., 25, 2469-2472.

Webb, D. F., Cliver, E. W., Crooker, N. U., St. Cyr, O. C., Thompson, B. J. 2000. The relationship of halo CMEs, magnetic clouds and magnetic storms. J. Geophys. Res., in press.

Zarro, D. M., Sterling, A. C., Thompson, B. J., Hudson, H. S., Nitta, N. 1999. SOHO EIT observations of extreme-ultraviolet "dimming" associated with a halo coronal mass ejection. Astrophys. J., 520, L139-L142.

\section{Captions}

Figure 1. Views of the probable CME source region for the halo CME of 1997 April 7, in soft X-rays from SXT on Yohkoh. (a) Before eruption a sigmoid pattern is prominent. (b) After eruption a cusp structure is prominent, and the sigmoid structure is not visible. (c) Difference image, formed by subtracting Panel (a) from Panel (b); mass lost from the dark areas - referred to as dimming regions - is suspected to have been expelled as part of the CME. Panels in this figure are $312 " \times 312 "$ in size.

Figure 2. Images in soft X-rays from SXT ( $a$ and b), and EIT (c and d) from before (a and c) and after (b and d) a halo CME of 1996 December 19. Prior to the event a sigmoid feature is prominent in SXT, but not apparent in EIT. After the event a system of bright arcade loops has a manifestation in both SXT and EIT images. For these panels, the axes give the distance scale is in arcseconds with $(0,0)$ located at Sun center.

Figure 3. A schematic derived from recent observations of sigmoid structures related to CMEs (Sterling et al. 2000). Before eruption a sigmoid structure straddles a magnetic neutral line. After eruption the sigmoid structure is much fainter or absent, a bright arcade running normal to the neutral line brightens in SXT and EIT images, and a cusp is often visible in SXT images. Dashed lines in (a) indicate field lines running normal to the neutral line that are often not visible in SXRs or EUV prior to eruption. They are, however, seen prior to eruption in some cases (Moore \& LaBonte 1980). 


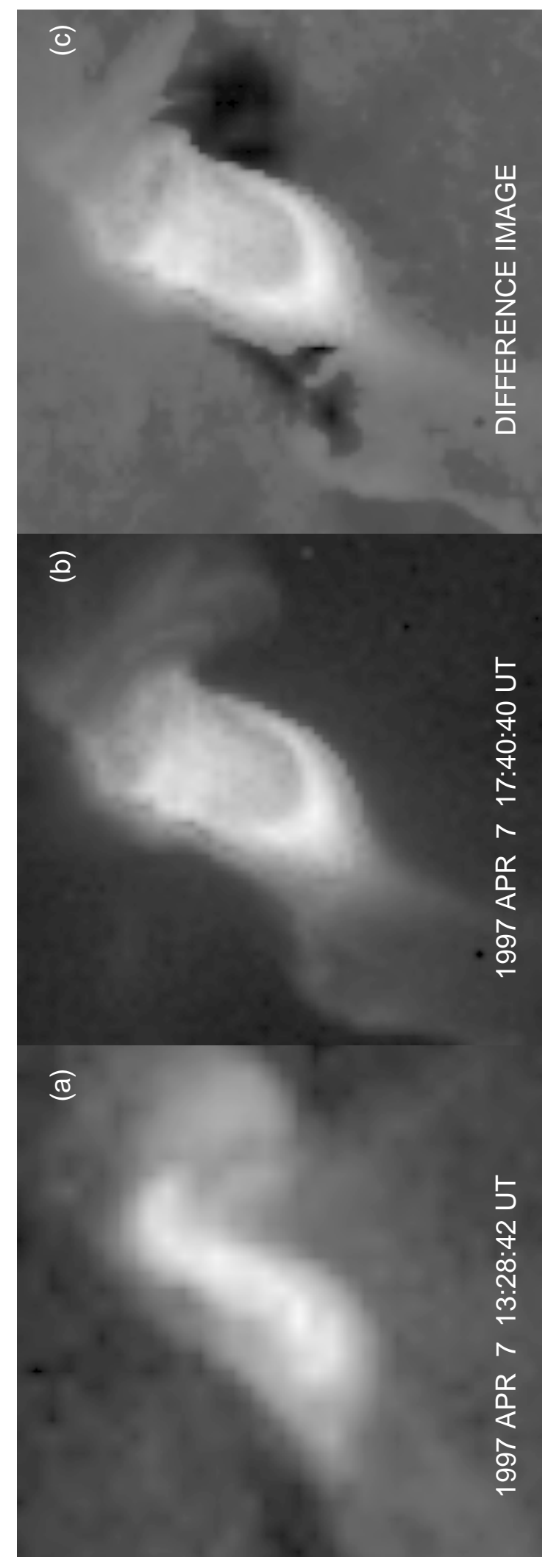

Figure 1: 

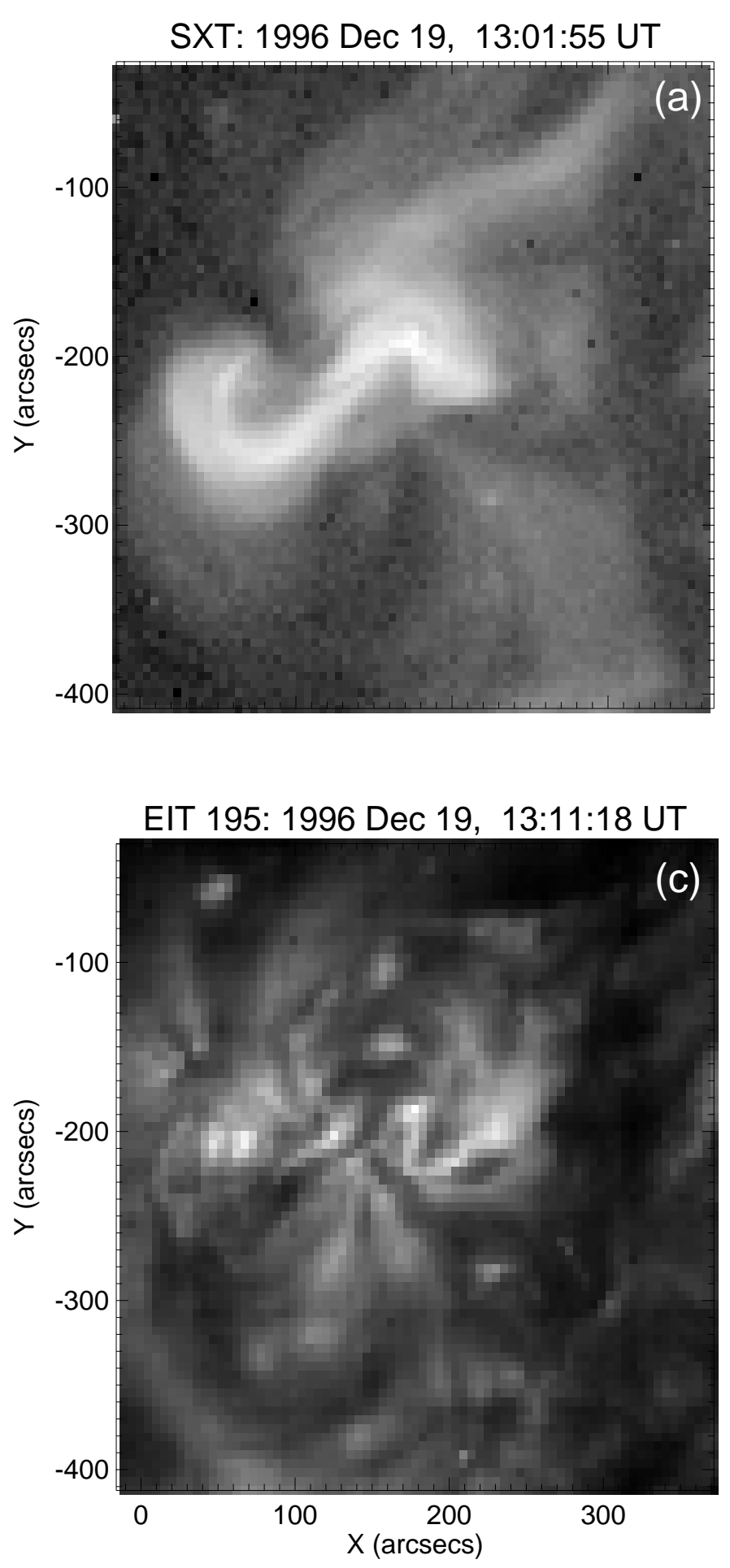

SXT: 1996 Dec 19, 17:11:09 UT

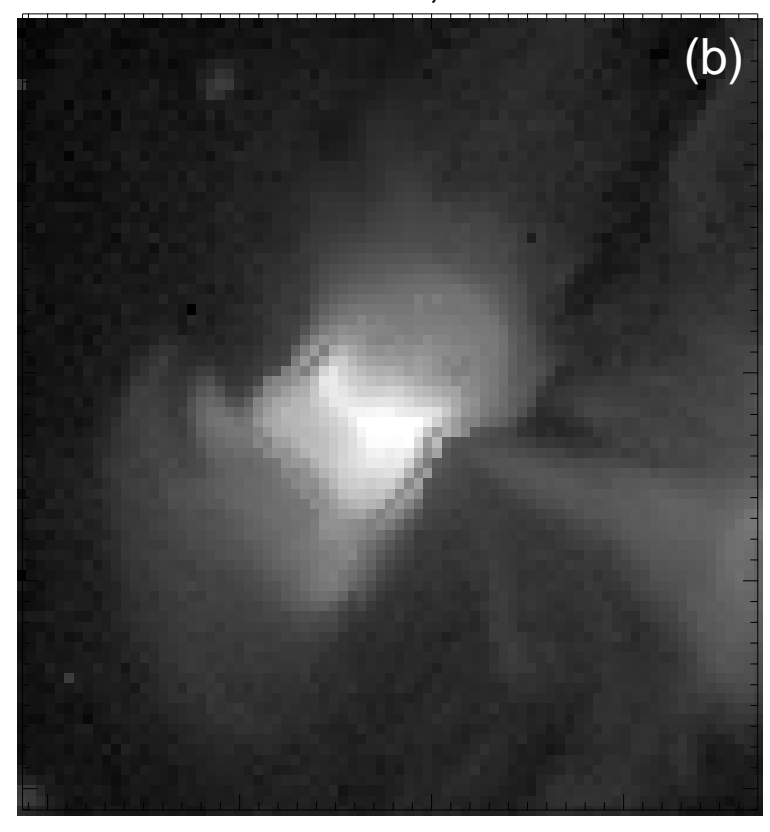

EIT 195: 1996 Dec 19, 21:03:35 UT

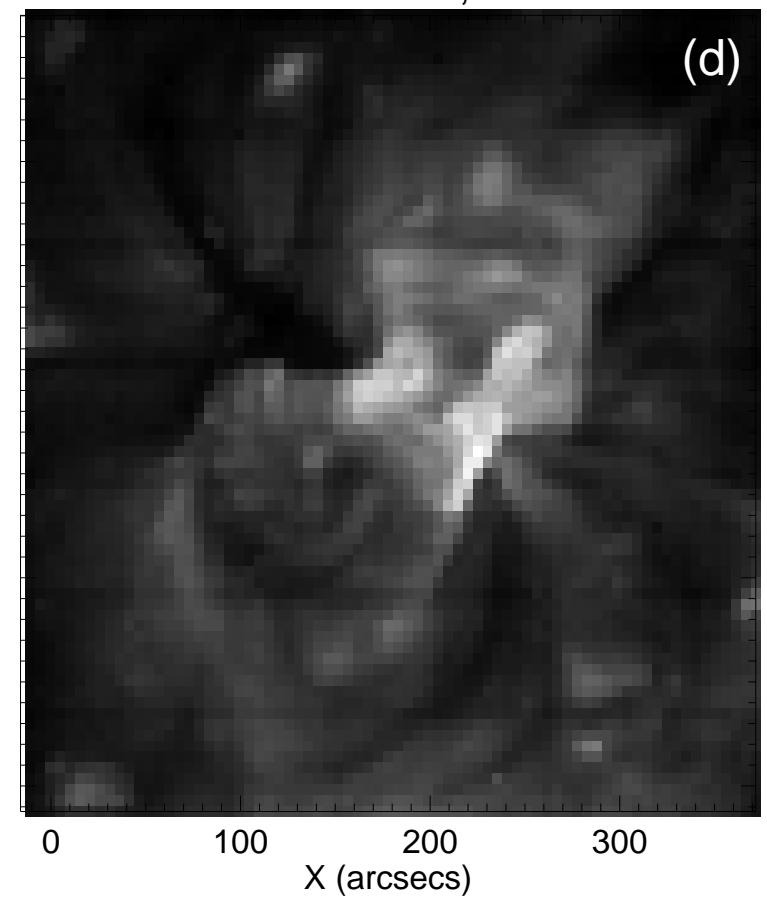

Figure 2: 


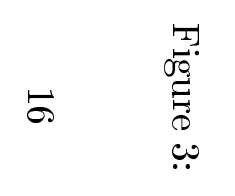

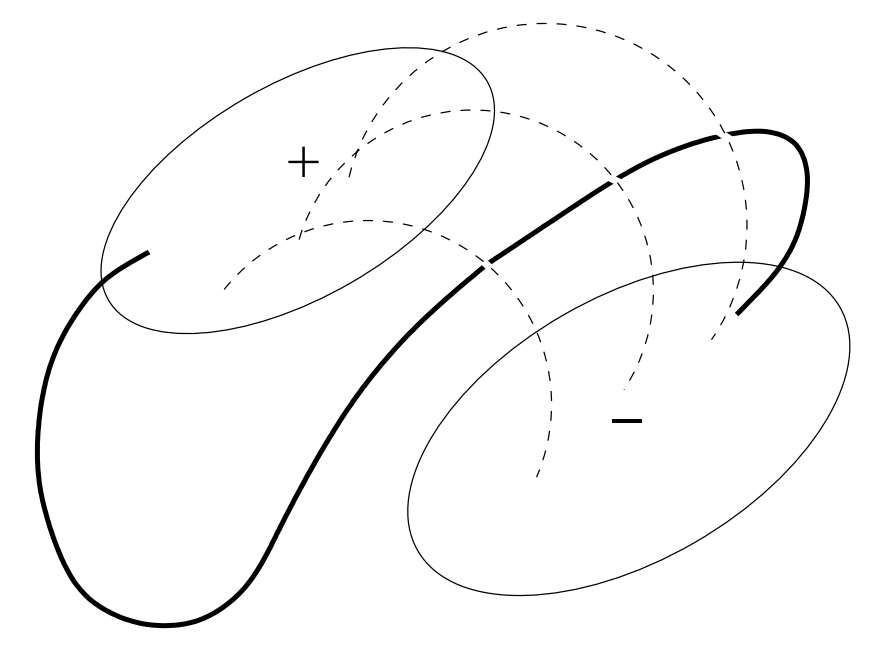

(a) Before

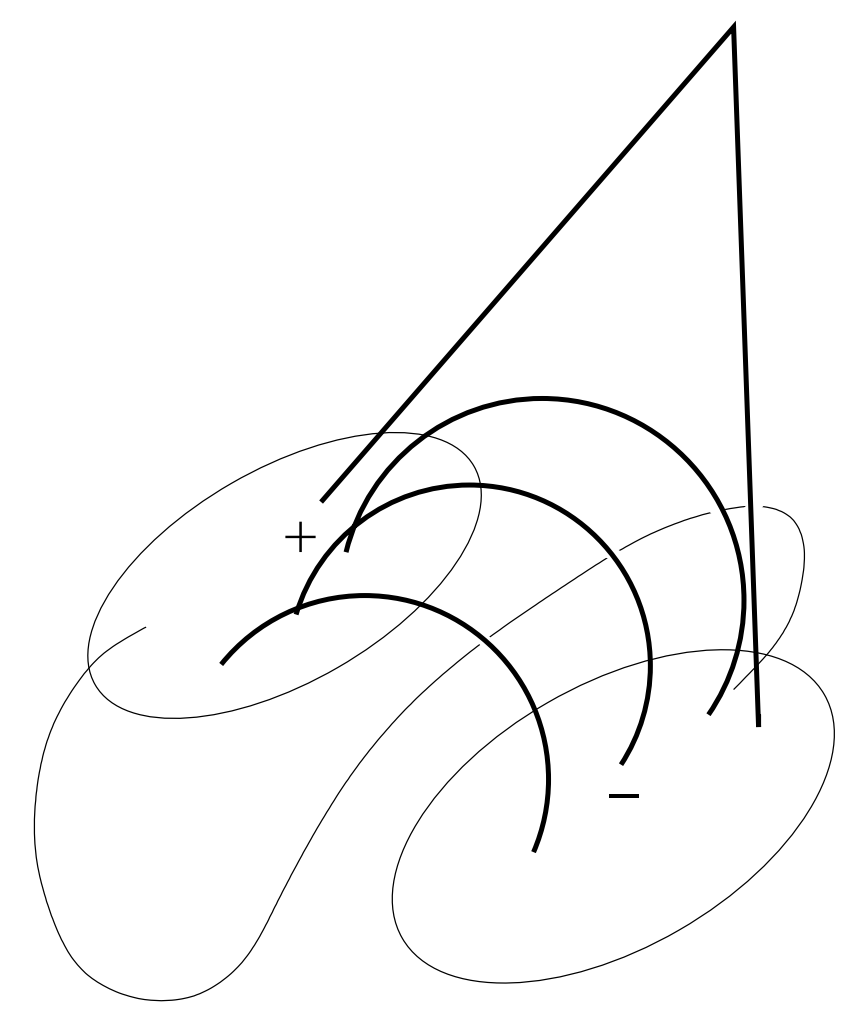

(b) After 